\title{
White Matter Microstructural Similarity and Diversity of Functional Constipation and Constipation- predominant Irritable Bowel Syndrome
}

\author{
Jiaofen Nan, ${ }^{1}$ Liangliang Zhang, ${ }^{1}$ Qiqiang Chen, ${ }^{1}$ Nannan Zong, ${ }^{1}$ Peiyong Zhang, ${ }^{2}$ Xing Ji, ${ }^{3}$ Shaohui Ma, ${ }^{4}$ Yuchen Zhang, ${ }^{4}$ \\ Wei Huang, ${ }^{1}$ Zhongzhou Du, ${ }^{1}$ Yongquan Xia, ${ }^{1}$ and Ming Zhang ${ }^{4 *}$ \\ ${ }^{1}$ School of Computer and Communication Engineering, Zhengzhou University of Light Industry, Zhengzhou, China; ${ }^{2}$ First Affiliated Hospital \\ of Xinxiang Medical University, Weihui, China; ${ }^{3}$ First Affiliated Hospital of Yan'an University, Yan'an, China; and ${ }^{4}$ Department of Medical \\ Imaging, First Affiliated Hospital of Xi'an Jiaotong University, Xi'an, China
}

\section{Background/Aims}

The Rome III criteria separated chronic constipation into functional constipation (FC) and constipation-predominant irritable bowel syndrome (IBS-C), but some researchers questioned the partitioning and treated both as distinct parts of a continuum. The study aims to explore the similarity and diversity of brain white matter between FC and IBS-C.

\section{Methods}

The voxel-wise analysis of the diffusion parameters was used to quantify the white matter changes of female brains in $18 \mathrm{FC}$ patients and 20 IBS-C patients compared with a comparison group with 19 healthy controls by tract-based spatial statistics. The correlations between diffusive parameters and clinical symptoms were evaluated using a Pearson's correlation.

\section{Results}

In comparison to healthy controls, FC patients showed a decrease of fractional anisotropy (FA) and an increase of radial diffusivity (RD) in multiple major fibers encompassing the corpus callosum (CC, $P=0.001$ at peak), external capsule $(P=0.002$ at peak), corona radiata (CR, $P=0.001$ at peak), and superior longitudinal fasciculus (SLF, $P=0.002$ at peak). In contrast, IBS-C patients showed FA and RD aberrations in the CC ( $P=0.048$ at peak). Moreover, the direct comparison between $\mathrm{FC}$ and IBS-C showed only RD differences in the CR and SLF. In addition, FA and RD in the CC were significantly associated with abdominal pain for all patients, whereas FA in CR $(P=0.016)$ and SLF $(P=0.040)$ were significantly associated with the length of time per attempt and incomplete evacuation separately for FC patients.

\section{Conclusion}

These results may improve our understanding of the pathophysiological mechanisms underlying different types of constipation.

(J Neurogastroenterol Motil 2018;24:107-118)

Key Words

Brain; Diffusion tensor imaging; Irritable bowel syndrome

Received: March 17, 2017 Revised: May 24, 2017 Accepted: August 16, 2017

(.) This is an Open Access article distributed under the terms of the Creative Commons Attribution Non-Commercial License (http://creativecommons. org/licenses/by-nc/4.0) which permits unrestricted non-commercial use, distribution, and reproduction in any medium, provided the original work is properly cited.

${ }^{*}$ Correspondence: Ming Zhang, MD

Department of Medical Imaging, First Affiliated Hospital of Xi'an Jiaotong University, No. 277, West Yanta Road, Xi'an 710061, China Tel: +86-29-82657695, Fax: +86-29-82657695, E-mail: profzmmri@gmail.com 


\section{Introduction}

Chronic constipation is a common clinical syndrome characterized by recurrent symptoms including reduced frequency of defecation, lumpy or hard stools, feeling of incomplete defecation, straining, obstructed defecation, and need for digital facilitation of defecation. ${ }^{1}$ Along with the change of lifestyle, global prevalence of chronic constipation showed an increasing trend year by year. Constipation patients often experience impaired quality of life and increased absences from work, which cause a heavy burden on patients, physicians, and the healthcare system. Despite different therapies for chronic constipation, multiple patients are dissatisfied with the therapeutic effects. The current results are attributed to unclear standards and pathophysiology behind chronic constipation. Over the years, the Rome committee has been working to standardize the definition of constipation. In the most recent Rome III criteria, they separated chronic constipation into functional constipation (FC) and constipation-predominant irritable bowel syndrome (IBSC) ${ }^{1-3}$ Some authors questioned the partitioning and treated the FC and IBS-C as distinct parts of a continuum, with differences based only on symptom severity. ${ }^{4-8}$ Nevertheless, differential responses to treatment seem to suggest that FC and IBS-C may be different disorders. ${ }^{9}$ Whether being different points of the same disorders or distinct disorders, it is of great importance in illustrating pathophysiological mechanisms underlying the discrimination between FC and IBS-C. However, the data supporting different etiologies between FC and IBS-C are scant.

A significant number of published studies have attributed the symptoms of functional gastrointestinal disorders (FGIDs) to dysregulation of the brain-gut axis $(\mathrm{BGA})^{10-16}$ that reflects a framework account for bidirectional interactions between the central nervous system (CNS), autonomic nervous system (ANS), and enteric nervous system (ENS). Within the framework, the CNS integrates communication within the BGA, thereby enabling the CNS to regulate the ENS via sympathetic and parasympathetic pathways. ${ }^{12}$ The ENS in turn works through local excitatory and inhibitory motor neurons that release neurotransmitters. ${ }^{11}$ As 2 types of FGIDs, $\mathrm{FC}$, and IBS-C have also been demonstrated to be associated with BGA disorders, in which the brain neural system was reported to have momentous functions. ${ }^{10-13}$ Therefore, CNS studies could make it easier to understand the differentiation between $\mathrm{FC}$ and IBS-C.

Recently, the rapid advancement of neuroimaging techniques made it convenient to investigate CNS mechanisms underlying various diseases including IBS and FC. Weaver et $\mathrm{al}^{10}$ summarized 27 recent studies that used neuroimaging techniques to explore etiologies of IBS patients, primarily including resting-state functional magnetic resonance imaging (rs-fMRI), ${ }^{17-20}$ fMRI during rectal distension, ${ }^{21-29}$ structural MRI, ${ }^{30-36}$ and diffusion tensor imaging (DTI). ${ }^{37-39}$ Across rs-fMRI studies, consistency was observed in the insula with increased patterns. Brain response to rectal distension revealed that the most consistently activated regions were in the insula, anterior cingulate cortex, amygdala, middle cingulate cortex and prefrontal cortex. Structural MRI investigations reported the most consistent results with a reduction of the cortical thickness in the anterior insula and an increase of cortical thickness/grey matter volume in the postcentral gyrus. With regard to DTI investigations, only 3 works focused on IBS and reported inconsistent results. ${ }^{37-39}$ Chen et $\mathrm{al}^{37}$ extracted regions of interest (ROIs) based on fMRI studies and used DTI to detect the fractional anisotropy (FA) values of the ROIs. They found higher FA in both the fornix and external capsule (EC), providing preliminary evidence for white matter (WM) alterations in IBS patients. Ellingson et $\mathrm{a}^{38}$ investigated WM regions that were segmented using an FA threshold of 0.3 and performed probabilistic tractography based on targeted ROIs. The results exhibited lower FA in the basal ganglia, thalamic regions and sensory/motor association/integration regions as well as higher FA in the frontal lobe regions and corpus callosum (CC) in IBS patients. Moreover, patients had higher mean diffusivity (MD) in the thalamus, internal capsule (IC), and corona radiata (CR) as well as lower MD within the globus pallidus. The above both studies about white matter had arbitrariness and subjectivity in the choice of ROIs. Fang et $\mathrm{al}^{39}$ investigated voxel-wise WM changes in IBS patients, which overcame limitations encountered with the ROI-based method. They found that patients had significantly reduced $\mathrm{FA}$ in the right superior $\mathrm{CR}$, the splenium of the $\mathrm{CC}$ and the right retrolenticular area of the IC as well as increased $\mathrm{MD}$ in the right posterior limb and retrolenticular area of the IC, the right superior $\mathrm{CR}$ and the splenium and body of the $\mathrm{CC}$. In addition, they also found significantly increased axial diffusivity $(\mathrm{AD})$ in the bilateral retrolenticular area of the IC, the left posterior limb of the IC and the splenium of the CC. As compared to IBS, there is a small number of brain imaging studies on FC. As far as we know, only one study investigated the neural mechanism underlying $\mathrm{FC} .^{13}$ Zhu et $\mathrm{al}^{13}$ adopted rs-fMRI in conjunction with the amplitude of low frequency fluctuation analysis and Granger causality analysis to investigate the alterations of basal brain activity in FC patients. They found significant differences in a number of major brain regions implicated in somatic and sensory processing, and emotional 
modulation. The functional abnormalities at the basal level revealed that the brain-gut dysfunction may also impact FC. However, existing studies are still far from explaining the differences between FC and IBS-C, which may lead to not specifically targeting patients with different types of constipation.

In this study, DTI was utilized to obtain quantitative maps of diffusion parameters such as $\mathrm{FA}$, radial diffusivity $(\mathrm{RD}), \mathrm{AD}$, and $\mathrm{MD}$, and then assess the WM microstructural organization and integrity of the brain. The aim of the study is to investigate the similarity and diversity between 2 types of constipation (FC and IBS-C). The investigation will improve our understanding of the pathological mechanism underlying chronic constipation.

\section{Materials and Methods}

Prior to scanning, subjects provided written informed consent approved by the Institutional Review Board of the First Affiliated Hospital of the Medical College in Xi'an Jiaotong University (2013KL011).

\section{Subjects}

Since the female gender and younger age are risk factors for IBS, we recruited women as constipation patients and healthy controls (HC) in the age range of 18 to 30 years. A digestive clinician enlisted and interviewed constipation volunteers and non-constipation controls according to the criteria of chronic constipation without organic abnormalities. After the first culling, the involved subjects were re-evaluated.

Patients who stayed behind met the IBS-C and FC screening guidelines of the Rome III diagnostic criteria. ${ }^{1}$ IBS-C is diagnosed when defecation can relieve abdominal pain or discomfort, whereas the diagnosis of $\mathrm{FC}$ is made only if chronically constipated patients do not meet IBS criteria. Exclusion criteria were: (1) a history of drug abuse; (2) a history of loss of consciousness caused by various reasons; (3) gastric atrophy or ulcers; (4) erosive gastroduodenal lesions; (5) esophagitis; (6) neurological or psychiatric disorders; (7) respiratory, cardiovascular, renal, and hepatic diseases; (8) lactating or pregnant women; and (9) contraindication to MRI scanning. Of note, according to the Rome III diagnostic criteria, the 2 types of patients were mutually exclusive, and thus we excluded subjects that could not be diagnosed with FC or IBS-C deterministically. To avoid the effects of drugs, we also excluded those under active medical care for gastrointestinal complaints or taking regular medication.

\section{Clinical Questionnaires}

All participants completed the commonly used measures for constipated symptoms, simplified Constipation Scoring System (CSS), an 8-item inventory including painful evacuation, abdominal pain, incomplete evacuation, frequency of bowel movements, assistance for evacuation, unsuccessful attempts for evacuation per 24 hours, length of time per attempt, and duration of constipation. ${ }^{40}$ Each item is graded on a scoring range of 0 to 4 except for the item "assistance for defecation" (range from 0 to 2). Therefore, the range of the total score is 0 to 30 .

The quality of life was measured by the Short-Form Health Survey with 8 dimensions including 4 physical subscales (physical functioning, role physical, bodily pain, and general health), and 4 psychological subscales (vitality, social functioning, role emotional, and mental health). ${ }^{41}$ Two higher-order summary scores can be computed based on the appropriate weighted sum of the above subscale scores: mental component summary (MCS) and physical component summary (PCS). MCS is obtained by positively weighing each of the psychological subscales and negatively weighing each of the physical subscales. Conversely, PCS is calculated by positively weighing each of the physical subscales and negatively weighing each of the psychological subscales.

Anxiety and depression were measured using the 20-item selfrating anxiety scale (SAS) ${ }^{42}$ and self-rating depression scale (SDS) ${ }^{43}$ separately (scores ranging on a scale from 1-4 for each item). Their total scores were in the range of $20-80$, normalized to the standard scores by multiplying them by 1.25 . Therefore, the scores of SAS and SDS ranged from 25-100.

The scores of all questionnaires were from the average of 3 time points: the day of screening, a day before MRI scanning, and the day of MRI scanning. Good reliability and validity of these questionnaires for the Chinese population have been confirmed by Cronbach's alpha coefficients, test-retest reliability and Pearson's correlation coefficient. ${ }^{41,44-46}$

\section{Imaging Acquirement}

We implemented the experiment based upon a 3-T Signa GE MRI scanner (GE Healthcare, Milwaukee, WI, USA), and did not conduct scanning during the menstrual period to avoid experimental variation of the results. The experiment collected diffusion-weighted images (single-shot echo planar sequence) with 30 equidistant diffusion sensitizing directions $\left(b=1000 \mathrm{sec} / \mathrm{mm}^{2}\right.$ ) and a single $b=0 \mathrm{sec} / \mathrm{mm}^{2}$ image with the following parameters: repetition time/echo time $=6800 \mathrm{msec} / 93 \mathrm{msec}$; field of view $=$ 
$240 \mathrm{~mm}$; matrix $=128 \times 128$; and 45 slices with a $3 \mathrm{~mm}$ thickness and no gap. Diffusion-weighted images were collected again using the same acquisition scheme with the same parameters.

\section{Statistical Methods}

After averaging 2 acquisition images, the affine transformation with 12-degrees of freedom was utilized to perform the correction for eddy currents and head motion using FMRIB software library (FSL; FMRIB, Oxford, UK) (http://fsl.fmrib.ox.ac.uk/fsl/). Subsequently, voxel-wise WM analyses were performed using the Track-based Spatial Statistics (TBSS) package (FMRIB) (http:// fsl.fmrib.ox.ac.uk/fsl/tbss/index.html). ${ }^{47}$ Briefly, the corrected volume was employed to construct the voxel-wise diffusion tensor. The eigenvalues and eigenvectors were computed from the constructed $3 \times 3$ diffusion tensor matrix followed by the acquisition of $\mathrm{FA}$, $\mathrm{MD}, \mathrm{RD}$, and AD. Next, each subject's FA images underwent linear affine transformation and then elastic registration to align to the JHU ICBM-DTI-81 FA atlas in FSL. The aligned FA images for all subjects were averaged and then thinned to construct a skeletonized image of WM using an FA threshold of 0.2. Next, the linear and then the non-linear aligned FA image of each subject was projected onto the skeletonized image. In addition, the linear and then the nonlinear transformation matrices were also applied to the other metrics (MD, RD, and $\mathrm{AD}$ ). Once voxel-wise $\mathrm{MD}, \mathrm{RD}$, and $\mathrm{AD}$ maps were aligned to the same atlas, these non-FA parameters were also projected onto the FA skeletonized image. Finally, group differences for $\mathrm{FA}, \mathrm{MD}, \mathrm{RD}$, and $\mathrm{AD}$ maps were explored using non-parametric permutation testing based upon a voxel-wise $t$ test with 5000 random permutations using the randomize function. Threshold-free cluster enhancement (TFCE) was performed based on the statistical results for the multiple comparison correction. Significantly different clusters $(P<0.05$ after the correction of threshold-free cluster enhancement) were enlarged using the tbss fill function and were superimposed on the ICBM-DTI-81 WM atlas for visualization. ROIs were created from the WM intergroup differences between $\mathrm{HC}$ and patients with constipation in diffusivity parameters. Pearson's correlation analyses were utilized to assess the relationships between mean DTI measurements for ROIs and the subscales of CSS, PCS, MCS, SAS, and SDS.

Table. Demographic Statistics of Study Samples

\begin{tabular}{|c|c|c|c|c|c|}
\hline Items & Subscales & $\mathrm{HC}(\mathrm{n}=19)$ & $\mathrm{FC}(\mathrm{n}=18)$ & IBS-C $(\mathrm{n}=20)$ & $P$-value \\
\hline Age (yr) & - & $22.74 \pm 1.19$ & $21.11 \pm 1.28$ & $21.9 \pm 1.41$ & $>0.05$ \\
\hline Weight (kg) & - & $53 \pm 7.29$ & $50.39 \pm 6.16$ & $50.95 \pm 4.22$ & $>0.05$ \\
\hline Height (cm) & - & $161.16 \pm 6.56$ & $158.89 \pm 4.8$ & $157.5 \pm 4.95$ & $>0.05$ \\
\hline Duration (mo) & - & - & $61.83 \pm 36.28$ & $66.1 \pm 27.68$ & $>0.05$ \\
\hline \multirow[t]{9}{*}{ CSS } & Frequency of bowel movements & - & $0.67 \pm 0.59$ & $1 \pm 0.73$ & $>0.05$ \\
\hline & Painful evacuation & - & $3.22 \pm 0.65$ & $3.3 \pm 0.66$ & $>0.05$ \\
\hline & Incomplete evacuation & - & $2.89 \pm 0.76$ & $3.3 \pm 0.73$ & $>0.05$ \\
\hline & Abdominal pain & - & $1.56 \pm 0.51$ & $2.7 \pm 0.67$ & $<0.0001^{\mathrm{a}}$ \\
\hline & Length of time per attempt & - & $1.83 \pm 0.92$ & $2.2 \pm 1.20$ & $>0.05$ \\
\hline & Assistance for defecation & - & $0.22 \pm 0.43$ & $0.35 \pm 0.49$ & $>0.05$ \\
\hline & Unsuccessful attempts for evacuation per 24 hours & - & $0.83 \pm 0.38$ & $0.95 \pm 0.51$ & $>0.05$ \\
\hline & Duration of constipation & - & $1.44 \pm 0.70$ & $1.75 \pm 0.64$ & $>0.05$ \\
\hline & Total score & - & $12.67 \pm 2.45$ & $15.55 \pm 3.03$ & $<0.005^{\mathrm{a}}$ \\
\hline \multirow[t]{2}{*}{ SF-36 } & PCS & $57.21 \pm 1.73$ & $56.53 \pm 3.83$ & $55.11 \pm 5.22$ & $>0.05$ \\
\hline & MCS & $55.9 \pm 2.99$ & $44.68 \pm 9.08$ & $44.20 \pm 11.04$ & $<0.0001^{\mathrm{b}, \mathrm{c}}$ \\
\hline SAS & - & $29.54 \pm 4.13$ & $37.71 \pm 5.85$ & $40.88 \pm 6.13$ & $<0.0001^{\mathrm{b}, \mathrm{c}}$ \\
\hline SDS & - & $27.89 \pm 4.02$ & $41.04 \pm 7.22$ & $39.81 \pm 7.61$ & $<0.0001^{\mathrm{b}, \mathrm{c}}$ \\
\hline
\end{tabular}

${ }^{a}$ Indicates the presence of a significant difference between functional constipation (FC) and constipation-predominant irritable bowel syndrome (IBS-C) at a given threshold.

${ }^{\mathrm{b}}$ Indicates the presence of a significant difference between healthy controls (HC) and $\mathrm{FC}$ at a given threshold.

'Indicates the presence of a significant difference between $\mathrm{HC}$ and IBS-C at a given threshold.

CSS, Constipation Scoring System; SF-36, Short-Form Health Survey; SAS, self-rating anxiety scale; SDS, self-rating depression scale; PCS, physical component summary; MCS, mental component summary.

Data are presented as mean $\pm \mathrm{SD}$. 
$\mathrm{SAS} ; P=0.614$ for $\mathrm{SDS})$.

\section{Results}

\section{Clinical Results}

The study included $18 \mathrm{FC}$ patients, 20 IBS-C patients, and a comparison group of $19 \mathrm{HC}$ (all women). The 3 groups (all female) did not differ in terms of age, weight, and height. Omnibus results showed highly significant differences among groups $(\mathrm{F}>$ 11) for $\operatorname{CSS}\left(P=4.549 \mathrm{e}^{-25}\right), \operatorname{MCS}\left(P=6.361 \mathrm{e}^{-5}\right)$, SAS $(P=$ $\left.8.919 \mathrm{e}^{-8}\right)$, and $\operatorname{SDS}\left(P=1.229 \mathrm{e}^{-8}\right)$. Post hoc comparisons for group differences survived after correction for multiple comparisons. The detailed information with demographic statistics and clinical characteristics is shown in Table.

For the 2 patient groups, only abdominal pain (subscale in CSS) and CSS showed significant group differences. In particular, the severity of abdominal pain and discomfort that the IBS-C patients experienced far exceeded that the $\mathrm{FC}$ patients experienced $(\mathrm{T}$ $\left.=-5.944, P=8.264 \mathrm{e}^{-7}\right)$. However, we did not find significantly different mental or psychological health states between the FC group and IBS-C group $(P=0.883$ for MCS; $P=0.113$ for

\section{Imaging Results}

\section{Patients versus healthy controls}

In comparison to $\mathrm{HC}$, constipated patients showed changes of the diffusivity measurements in $\mathrm{FA}, \mathrm{MD}$, and $\mathrm{RD}$ in nearly all major WM tracts (Fig. 1A-C). No significant group differences in AD were observed at the $P<0.05$ level after TFCE correction. The overlapping regions of the 3 indices for intergroup differences were located in the genu of the $\mathrm{CC}(P=0.048$ for FA; $P=0.022$ for MD; $P=0.028$ for RD), $\mathrm{EC}(P=0.046$ for $\mathrm{FA} ; P=0.020$ for $\mathrm{MD} ; P=0.012$ for $\mathrm{RD}), \mathrm{CR}(P=0.036$ for $\mathrm{FA} ; P=0.022$ for $\mathrm{MD} ; P=0.010$ for $\mathrm{RD})$, and superior longitudinal fasciculus (SLF, $P=0.035$ for FA; $P=0.016$ for MD; $P=0.008$ for RD) (Fig. 1D).

\section{Healthy controls versus functional constipation and healthy controls versus constipation-predominant irritable bowel syndrome}

After dividing patients into the FC and IBS-C group, they
A

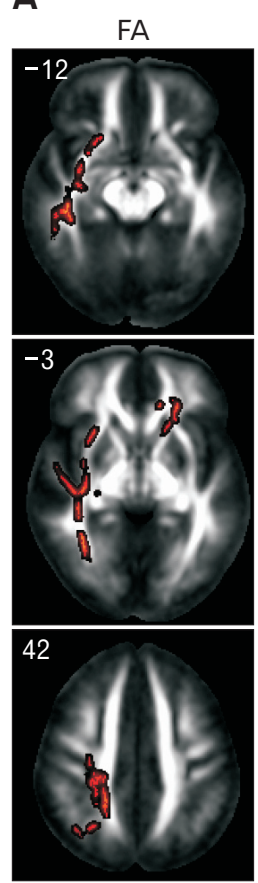

B

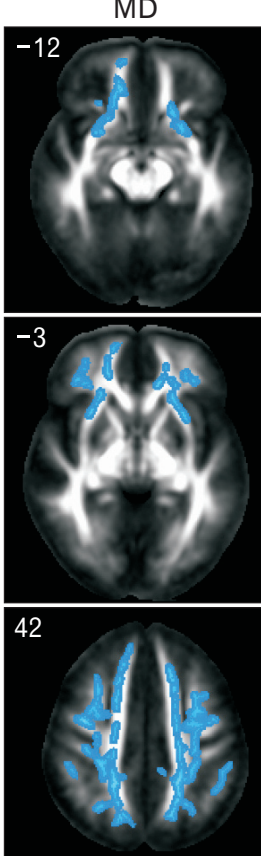

C

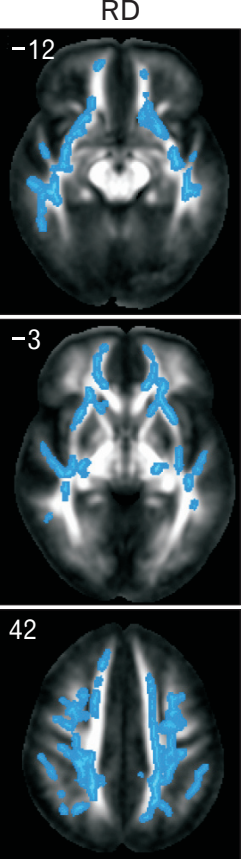

D

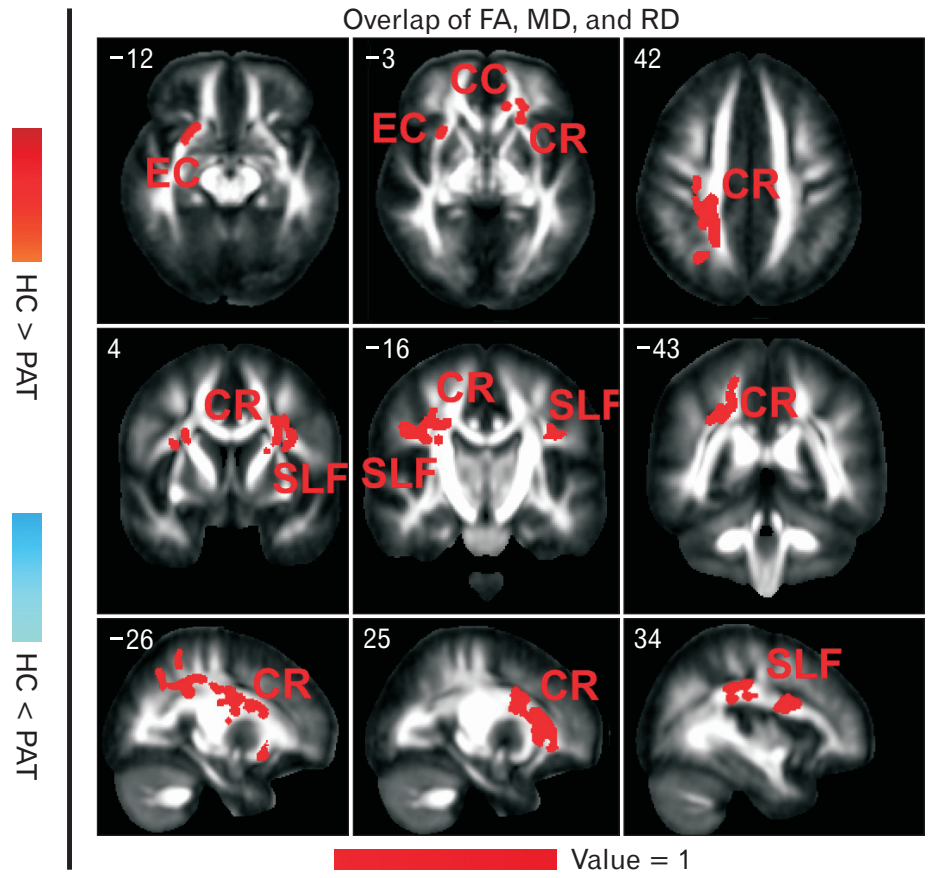

Figure 1. Changes in white matter parameters in constipated patients (PAT) compared with healthy controls (HC). (A) Changes of fractional anisotropy (FA) (the first column). (B) Changes of mean diffusivity (MD) (the second column). (C) Changes of radial diffusivity (RD) (the third column). (D) The overlap of the difference in FA, MD, and RD for patients vs HC. CC, corpus callosum; EC, external capsule; CR, corona radiata; SLF, superior longitudinal fasciculus. 
were compared separately with the $\mathrm{HC}$ group. With respect to $\mathrm{FC}$ patients, $\mathrm{FA}$ values were significantly reduced in numerous clusters encompassing the bundles of CC ( $P=0.008$ at peak), which connected interhemispheric cortices; the $\mathrm{CR}(P=0.018$ at peak) and SLF ( $P=0.014$ at peak), which subserved the frontal to temporalparietal-occipital connectivity; and the $\mathrm{EC}(P=0.010$ at peak) adjacent to the insula and the orbital part of the frontal gyrus (Fig. $2 \mathrm{~A})$. IBS-C patients exhibited a reduction of FA values only in the genu of the $\mathrm{CC}(P=0.049$ at peak $)$ that subserved the interhemispheric interaction (Fig. 2B).

Similar to FA, FC patients exhibited RD increases (Fig. 2A) in numerous clusters $(P=0.001$ for $\mathrm{CC} ; P=0.001$ for $\mathrm{CR} ; P=$ 0.002 for SLF; $P=0.002$ for EC), whereas IBS patients exhibited RD increases (Fig. 2B) only in the genu of the CC $(P=0.048$ at peak). The statistically altered regions in $\mathrm{FC}$ patients and those in IBS-C patients overlapped at the genu of the CC (Fig. 2C).
In contrast to $\mathrm{FA}$ and $\mathrm{RD}$, significant clusters of increased MD occurred only in the FC group, but the IBS-C group tended to have increased MD than the $\mathrm{HC}$ group without significance after correction. Therefore, Figure 3 showed the overlapping regions of 3 parameters (FA, MD, and $\mathrm{RD}$ ) for the $\mathrm{HC}$ group versus $\mathrm{FC}$ group (Fig. 3A) and the overlapping clusters of only 2 parameters (FA and RD) for the HC group versus IBS-C group (Fig. 3B).

\section{Functional constipation versus constipation-predominant irritable bowel syndrome}

We also tested group differences between the FC and IBS-C in $\mathrm{FA}, \mathrm{AD}, \mathrm{MD}$, and RD. Results showed the significant differences only in $\mathrm{RD}$, and $\mathrm{FC}$ patients had a greater RD than IBS-C patients in the CR and SLF (Fig. 3C).
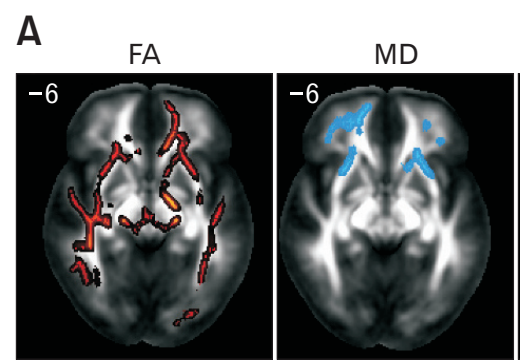

FC vs HC
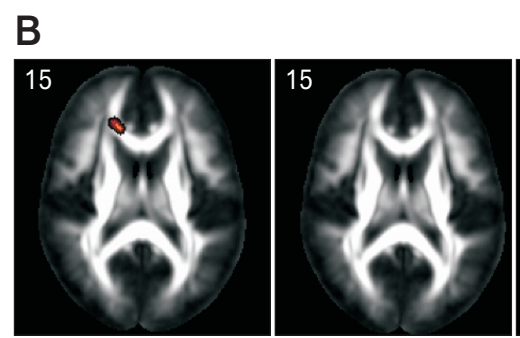

IBS-C vs HC

\section{C}
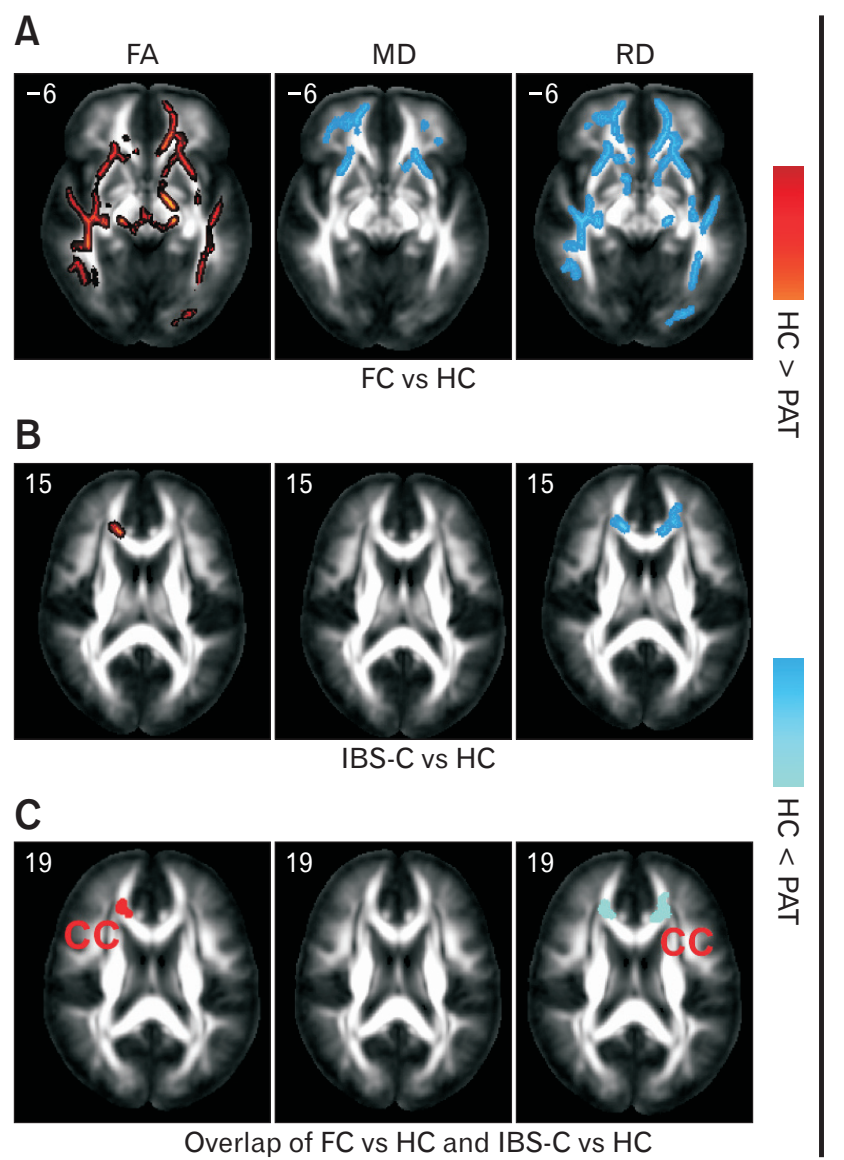

Overlap of FC vs HC and IBS-C vs HC
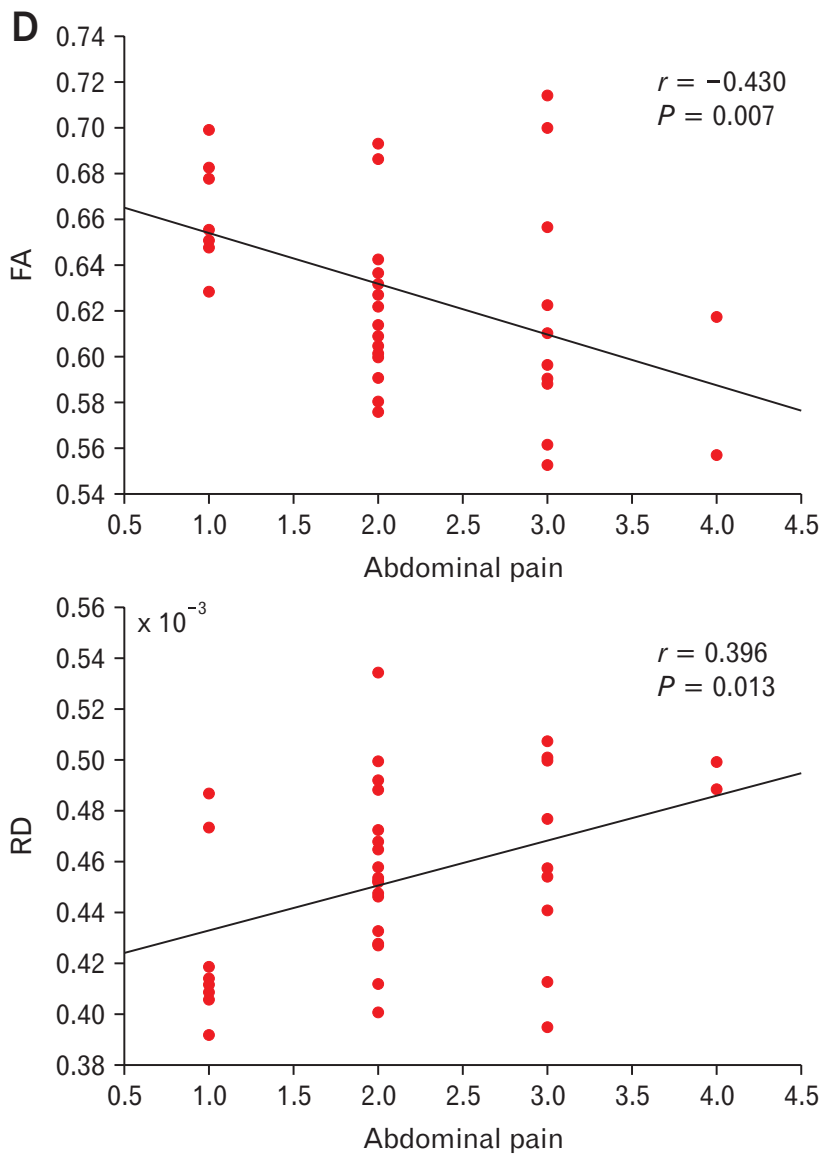

Figure 2. Changes of white matter in functional constipation (FC) and constipation-predominant irritable bowel syndrome (IBS-C) patients compared with healthy controls (HC). (A) FC vs HC (the first row). (B) IBS-C vs HC (the second row). (C) Overlap of FC vs HC and IBS-C vs HC. (D) Correlation between diffusivity parameters in the overlapping region (corpus callosum [CC]) and clinical symptoms. FA, fractional anisotropy; MD, mean diffusivity; $\mathrm{RD}$, radial diffusivity. 
A
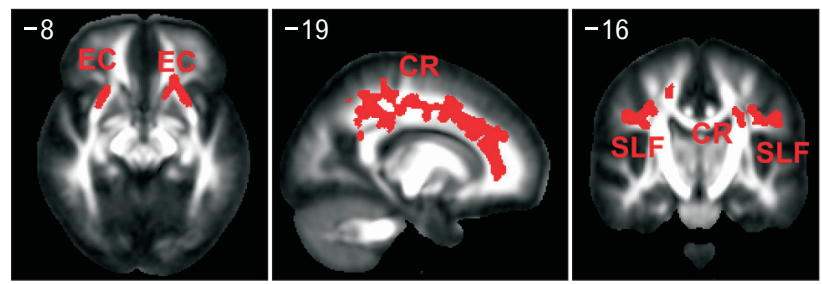

Overlap of FA, MD, and RD with FC vs HC

B
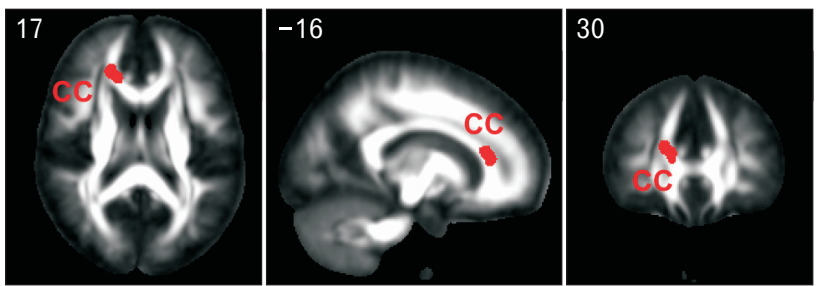

Overlap of FA and RD with IBS-C vs HC

\section{C}

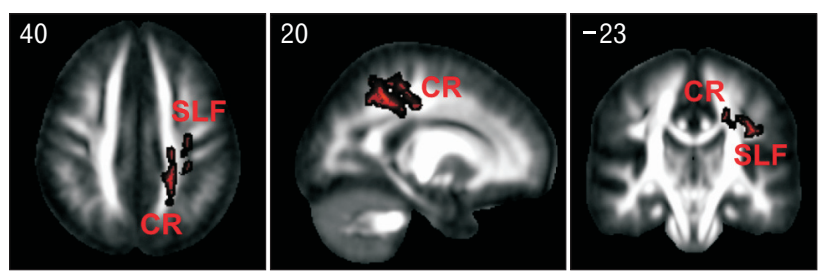

Difference of RD with FC vs IBS-C

Figure 3. Overlap of white matter parameters with differences between patients and healthy controls (HC). EC, external capsule; CR, corona radiata; SLF, superior longitudinal fasciculus; CC, corpus callosum; FA, fractional anisotropy; MD, mean diffusivity; RD, radial diffusivity; FC, functional constipation; IBS-C, constipationpredominant irritable bowel syndrome.

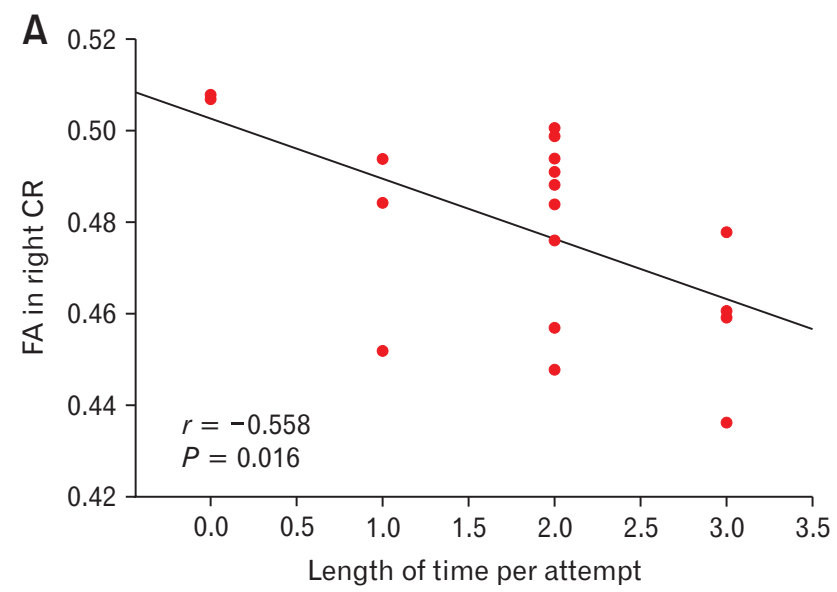

\section{Correlation Analysis}

The mean $\mathrm{FA}$ in the $\mathrm{CC}$ that showed a significant decrease in both groups of patients relative to $\mathrm{HC}$ (Fig. 2C) was inversely correlated with the abdominal pain score of the $\operatorname{CSS}(r=-0.430, P=$ 0.007), as shown in Figure 2D. The voxel-wise average of the RD that showed a significant increase in both groups of patients relative to $\mathrm{HC}$ (Fig. 2C) positively correlated with the abdominal pain score ( $r=0.396, P=0.013$ ) for all constipated patients (Fig. 2D).

Additionally, significant correlations were examined between imaging measurements (especially FA) in significantly different clusters (FC vs $\mathrm{HC}$ or IBS vs $\mathrm{HC}$ ) and clinical variables. Consequently, we found an adversely proportional relationship $(P=$ 0.016) between FA in the CR and the length of time per attempt and a positive correlation $(P=0.040)$ between FA in the SLF and feeling of incomplete evacuation for the FC group (Fig. 4A and 4B). Nevertheless, no significant correlation was found in the IBSC group.

\section{Discussion}

The present study aimed to investigate the similarity and diversity of female FC patients and female IBS-C patients. Primary results showed similar microstructural abnormalities in the genu of the $\mathrm{CC}$ for both groups with patients. Moreover, FC patients had more regions with microstructural abnormalities than IBS-C patients.

In the current study, we found reduced FA as well as increased $\mathrm{MD}$ and $\mathrm{RD}$ in patients as compared with $\mathrm{HC}$. FA is a measure of the anisotropic component of the diffusion tensor and evaluates

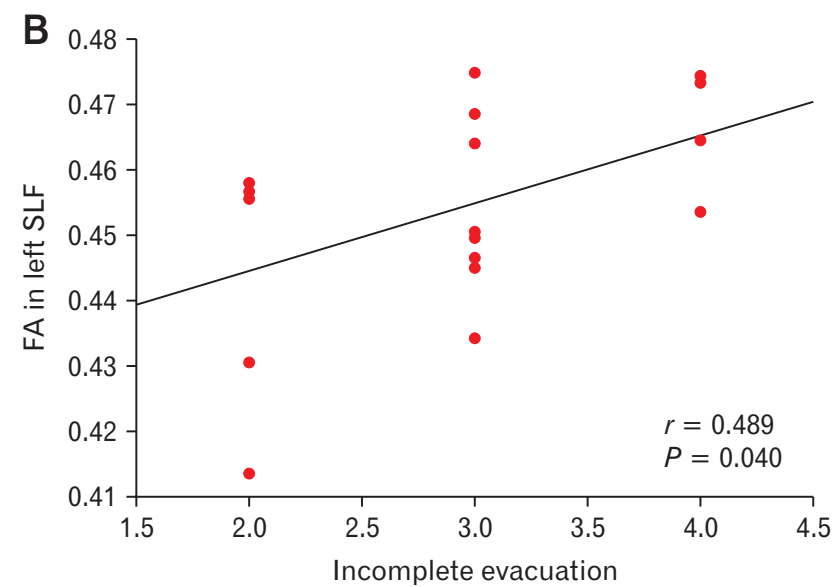

Figure 4. Correlation analysis between mean imaging parameters and clinical symptoms for patients with functional constipation. FA, fractional anisotropy; CR, corona radiata; SLF, superior longitudinal fasciculus. 
the degree to which diffusion prefers some direction over others. In WM, anisotropic diffusion is considered to be mainly caused by barriers imposed by intact membranes, and myelination could also modulate it. This is often interpreted to be a measure of WM integrity. It has been reported that a lower FA could usually be attributed to 3 possibilities: axonal injury, hypomyelination, and both comprehensive results. ${ }^{48,49} \mathrm{MD}$ represents the degree of diffusion but not the direction of diffusion, and it can reflect alterations in cell membranes and myelin integrity. Lower FA with higher MD of WM in the current results exist in the vast majority of neuropathologies. However, FA and MD are insufficient because they could not reflect specific reasons for damage to WM integrity (aberrant myelination, or altered axon density or diameter). AD and RD may provide further insight into these changes to some extent. AD represents the direction of axial diffusion, whereas $\mathrm{RD}$ represents the direction of radial diffusion. The former reflects the density and diameter of axons, while the latter is sensitive to the degree of myelination. In our study, there was no significant difference in AD of any WM tracts, but RD increased in the WM tracts along with a decrease in FA. These results might indicate hypomyelination and demyelination rather than changes in the axonal diameters or density, although axonal injury also influences RD. It might represent a decreased number of myelination fibers or a decreased ratio between longitudinal vs oblique aligned myelinated fibers. ${ }^{50,51}$

When exploring the central similarity of FC and IBS-C, the genu of the $\mathrm{CC}$ was found to be abnormal in both patient groups. Moreover, FA in the $\mathrm{CC}$ showed a negative association with abdominal pain or discomfort intensity, whereas $\mathrm{RD}$ in the $\mathrm{CC}$ positively correlated with pain or discomfort intensity. The results suggested that the $\mathrm{CC}$ disorder was associated with pain perception. The CC, as the largest fiber tract in the human brain, connects the 2 hemispheres of the brain. The main interhemisphere commissure is required for many aspects of neural function, encompassing inhibition of cortical functions, integration of sensory information, regulation of cognition, processing of emotion, and interhemispheric exchange of information. ${ }^{52,53}$ Multiple studies reported CC dysfunction in patients with chronic pain, such as fibromyalgia, ${ }^{54}$ migraine, ${ }^{55}$ and temporomandibular disorder, ${ }^{56}$ supporting the indicative role of the $\mathrm{CC}$ in pain perception and pain control. ${ }^{57}$ Therefore, we reasoned that aberrations in the WM integrity in the CC may cause the reduction of obstruction to water diffusion and reduced anisotropy, which can be responsible for the perceptional intensity of abdominal discomfort and pain in FC and IBS-C patients.

When considering the diversity, no symptoms except abdomi- nal pain intensity and CSS were found to be significantly different for both patient groups. As such, if they are two points of the same spectrum, owing to higher pain intensity and higher constipation score in IBS-C than in FC patients, IBS-C patients should have more regions or higher intensity in central abnormalities than FC patients. Amazingly, our finding was actually the opposite, and the FC exhibited far more regions in the central changes than IBS$\mathrm{C}$, especially in the CR and SLF (significantly different for FC vs IBS-C). CR includes projection fibers associated with the corticopontine tract, corticospinal tract and corticobulbar tract, which can radiate out from the cerebral cortex and then converge in the brain stem, and they can radiate out of the brain stem through the IC as well. Therefore, the brain stem is a vital structure of $\mathrm{CR}$ projections. A previous study showed that preganglionic motor neurons provide vagal innervations to the gastrointestinal tract and stem from the dorsal motor nucleus of the vagus nerve within the brainstem. ${ }^{12}$ Moreover, Lim et al reported that the colon transit time was delayed in patients with pontine lesions (a part of the brainstem). ${ }^{58}$ Therefore, we suspected that the aberrations in the CR associated with the corticopontine tract may be linked with CNS dysregulation of colon transit time in constipated patients. By comparing the colonic transit time of FC and IBS-C, Ansari et al found that wholegut transit time and rectosigmoid transit time tended to be slower in individuals with FC compared to individuals with IBS-C, ${ }^{59}$ which may explain why aberrations in the $\mathrm{CR}$ occurred in the $\mathrm{FC}$ group but not in the IBS-C groups. As a major association fiber, SLF connects the frontal lobe with the parietal lobe and temporoparietal junction area involving the transmission of somatosensory information, regulation of motor behavior and contribution to the corticocerebellar system. ${ }^{60}$ In the current results, we found that FA in the SLF was significantly associated with the incomplete defecation score that measures a feeling related to abnormal perception and/or failure of evacuation. In an earlier study, the "visceral motor" cortex was found to largely overlap the source of the fiber pathway from the medial part of the frontal cortex to the nucleus of the solitary tract. $^{61}$ This finding provided early evidence of a CNS pathway concerning gastric motility consistent with our speculation about $\mathrm{CR}$ associated with regulation of colon transit and SLF associated with perception and motor behavior. Therefore, the current result about differentiation in the $\mathrm{CR}$ and SLF between the FC group and IBS-C group is likely relevant to the delay of gastrointestinal transit time, aberration of abdominal perception, and descent of stomach motility in FC patients. Although the precise relationship between CNS aberrations and gastrointestinal transit time and motility in the FC group cannot be determined by this study without 
physiological tests, the current results about correlations (FA of the CR and the length of time per attempt; FA of the SLF and incomplete defecation) supports the above speculation. The speculation was also supported by the results reviewed by Siah et $\mathrm{al}^{62}$ that the pathophysiological mechanisms may distinguish FC from IBS including delayed colonic transit time.

Additionally, the importance of psychological and psychiatric factors should not be ignored. In the present study, we also detected differences in WM parameters after inclusion of SAS and SDS as covariates. Results were not changed significantly after controlling for SAS and SDS. Moreover, we failed to find a linear correlation between the SAS/SDS and DTI measures (in the CC: SAS-FA: $r$ $=-0.175, P=0.293$; SAS-RD: $r=0.104, P=0.533$; SDS-FA: $r=-0.159, P=0.342$; SDS-RD: $r=0.186, P=0.264)$. The above results seem to suggest that the observed WM differences were driven by gastrointestinal symptoms as opposed to psychological factors. To test this further, we performed a partial correlation between DTI parameters and gastrointestinal symptoms, and found a decrease in the linear correlation (FA-abdominal pain in the CC: $r=-0.398, P=0.016$; RD-abdominal pain in the CC: $r=0.388$, $P=0.019$ ) after controlling for SAS and SDS (Fig. 2D for the results before controlling for SAS and SDS). Moreover, the anxiety score (SAS) and abdominal pain score had a significant association $(r=0.335, P=0.039)$ based on the clinical data. These indicated that the influences of psychological factors on WM alterations may be reflected in the gastrointestinal symptoms. In the Chinese norm, ${ }^{63,64}$ SAS less than 50 and SDS less than 53 are considered to be in the normal range. In the current study, although patients had a significantly higher SAS and SDS than controls, only 2 subjects exhibited anxiety and 5 subjects exhibited depression. This may be a reason why we failed to detect the influences of psychiatric factors on WM alterations. Therefore, we suspected that the observed WM results may include an additive or synergistic relationship of psychological factors, which may be more much obvious over time.

Interestingly, the study by Fang et $\mathrm{al}^{39}$ examined WM abnormalities in IBS patients and found that these patients had significantly reduced $\mathrm{FA}$ and increased $\mathrm{MD}$ in the $\mathrm{IC}$ and $\mathrm{CR}$ as well as increased $\mathrm{AD}$ in the $\mathrm{IC}$ and $\mathrm{CC}$, which were completely different from our results. Clinically, this discrepancy may result from patients' conditions including age, gender, duration, type, anxiety/ depression symptoms, and gastrointestinal symptoms because of the consistent SPM analysis. To clarify the reason, we evaluated the clinical differences of subjects in the 2 studies. Because of limited clinical information from the study by Fang et al, ${ }^{39}$ we could compare the subjects' conditions only from the gender distribution, age and duration. There is no doubt that the gender distribution of participants was completely different in the two studies. More than half of the subjects were male in the study by Fang et al, ${ }^{39}$ while all subjects were female in the present study. Moreover, the subjects were noticeably older in the study by Fang et al ${ }^{39}$ than those in the current study. More importantly, all IBS patients from the present study belonged to constipation-predominant IBS, which was not introduced in the study by Fang et al. ${ }^{39}$ Therefore, we must interpret this with caution because of the lack of information, including anxiety, depression, severity of gastrointestinal symptoms and subtype of disease. We believe that the inconsistent results may be related to, but not restricted to the above items. Over the past decades, various theories have been used to explain the pathological mechanism underlying IBS, such as psychological dysfunction, ${ }^{65}$ visceral hypersensitivity ${ }^{66}$ and dysmotility, ${ }^{67}$ each of which is entwined with the nervous system. Given these factors, more well-thought-out studies are needed.

Several limitations should be noted in this study. First, the study contained a relatively small sample size, although group differences were detected at a relatively stringent threshold. The results need to be replicated in a large sample. Second, the research focused on gender- and age-specific subjects (females and 18-30 years old), thus the role of age and gender on WM microstructure in constipated patients is still an open question. While the sample size was underpowered to explore the central development with age, future research will aim to probe whether older patients show similar FA and $\mathrm{RD}$ alterations as those observed here. Finally, although the clinician involved in the work diagnosed subjects as FC, IBS-C or $\mathrm{HC}, \mathrm{FC}$, and IBS-C may transmute into each other according to a prior follow-up study. ${ }^{7}$ It appears likely that FC and IBS-C could not be reliably differentiated in a long-term relationship. Future ongoing efforts on follow-up studies with large samples of participants are warranted to reveal WM mechanisms underlying constipated patients in more detail.

In conclusion, on one hand, we observed a central similarity between FC and IBS-C for WM changes compared with HC, thus illustrating a possible mechanism of an overlap in constipation symptoms. On the other hand, although there were more serious symptoms in IBS-C patients than in FC patients, we found more central abnormalities in FC patients than in IBS-C patients, revealing the central diversity of both. These results seem to support that FC and IBS-C may have different pathophysiologies, which may boost pathophysiological understanding of patients with chronic constipation. If confirmed and extended by physiological tests and animal experiments, this may guide clinical practice for screening 
and intervention.

Financial support: This work was supported by the project for the National Natural Science Foundation of China (Grant No. 81501547, 61374014, 61602423 , 61501405, 81371530, and 81501548), and the Science and Technology Planning Program of Henan Province (Grant No. 172106000074, 172102210063, and 162102210218).

\section{Conflicts of interest: None.}

Author contributions: Jiaofen Nan: processing data, interpreting data, and drafting the article; Liangliang Zhang and Nannan Zong: processing data; Qiqiang Chen: processing data and interpreting data; Peiyong Zhang, Xing Ji, Shaohui Ma, and Yuchen Zhang: collecting data and analyzing data; Zhongzhou Du and Wei Huang: revising the manuscript; and Yongquan Xia and Ming Zhang: designing study and collecting data.

\section{References}

1. Drossman DA, Dumitrascu DL. Rome III: new standard for functional gastrointestinal disorders. J Gastrointestin Liver Dis 2006;15:237-241.

2. Drossman DA. The functional gastrointestinal disorders and the Rome III process. Gastroenterology 2006;130:1377-1390.

3. Longstreth GF, Thompson WG, Chey WD, Houghton LA, Mearin F, Spiller RC. Functional bowel disorders. Gastroenterology 2006;130:1480-1491.

4. Heidelbaugh JJ, Stelwagon M, Miller SA, Shea EP, Chey WD. The spectrum of constipation-predominant irritable bowel syndrome and chronic idiopathic constipation: US survey assessing symptoms, care seeking, and disease burden. Am J Gastroenterol 2015;110:580-587.

5. Shekhar C, Monaghan PJ, Morris J, et al. Rome III functional constipation and irritable bowel syndrome with constipation are similar disorders within a spectrum of sensitization, regulated by serotonin. Gastroenterology 2013;145:749-757.

6. Rey E, Balboa A, Mearin F. Chronic constipation, irritable bowel syndrome with constipation and constipation with pain/discomfort: similarities and differences. Am J Gastroenterol 2014;109:876-884.

7. Wong RK, Palsson OS, Turner MJ, et al. Inability of the Rome III criteria to distinguish functional constipation from constipation-subtype irritable bowel syndrome. Am J Gastroenterol 2010;105:2228-2234.

8. Zhao YF, Ma XQ, Wang R, et al. Epidemiology of functional constipation and comparison with constipation-predominant irritable bowel syndrome: the systematic investigation of gastrointestinal diseases in China (SILC). Aliment Pharm Ther 2011;34:1020-1029.

9. Enck P, Leinert J, Smid M, Köhler T, Schwille-Kiuntke J. Functional constipation and constipation-predominant irritable bowel syndrome in the general population: data from the GECCO study. Gastroenterology Res Pract 2016;2016:3186016.

10. Weaver KR, Sherwin LB, Walitt B, Melkus GD, Henderson WA. Neuroimaging the brain-gut axis in patients with irritable bowel syndrome. World J Gastrointest Pharmacol Ther 2016;7:320-333.

11. Harris LA, Hansel S, DiBaise J, Crowell MD. Irritable bowel syndrome and chronic constipation: emerging drugs, devices, and surgical treatments. Curr Gastroenterol Rep 2006;8:282-290.

12. Browning KN, Travagli RA. Central nervous system control of gastrointestinal motility and secretion and modulation of gastrointestinal functions. Compr Physiol 2014;4:1339-1368.

13. Zhu Q, Cai W, Zheng J, et al. Distinct resting-state brain activity in patients with functional constipation. Neurosci Lett 2016;632:141-146.

14. Nan J, Liu J, Mu J, et al. Brain-based correlations between psychological factors and functional dyspepsia. J Neurogastroenterol Motil 2015;21:103-110.

15. Nan J, Zhang L, Zhu F, et al. Topological alterations of the intrinsic brain network in patients with functional dyspepsia. J Neurogastroenterol Motil 2016;22:118.

16. Tanaka Y, Kanazawa M, Fukudo S, Drossman DA. Biopsychosocial model of irritable bowel syndrome. J Neurogastroenterol Motil 2011;17:131-139.

17. Gupta A, Kilpatrick L, Labus J, et al. Early adverse life events and resting state neural networks in patients with chronic abdominal pain: evidence for sex differences. Psychosom Med 2014;76:404-412.

18. Ma X, Li S, Tian J, et al. Altered brain spontaneous activity and connectivity network in irritable bowel syndrome patients: a resting-state fMRI study. Clin Neurophysiol 2015;126:1190-1197.

19. Hong JY, Kilpatrick LA, Labus J, et al. Patients with chronic visceral pain show sex-related alterations in intrinsic oscillations of the resting brain. J Neurosci 2013;33:11994-12002.

20. Hong JY, Kilpatrick LA, Labus JS, et al. Sex and disease-related alterations of anterior insula functional connectivity in chronic abdominal pain. J Neurosci 2014;34:14252-14259.

21. Hall GB, Kamath MV, Collins S, et al. Heightened central affective response to visceral sensations of pain and discomfort in IBS. Neurogastroenterol Motil 2010;22:276-e80.

22. Larsson MB, Tillisch $\mathrm{K}$, Craig $\mathrm{AD}$, et al. Brain responses to visceral stimuli reflect visceral sensitivity thresholds in patients with irritable bowel syndrome. Gastroenterology 2012;142:463-472, e3.

23. Elsenbruch S, Rosenberger C, Enck P, Forsting M, Schedlowski M, Gizewski ER. Affective disturbances modulate the neural processing of visceral pain stimuli in irritable bowel syndrome: an fMRI study. Gut 2010;59:489-495.

24. Icenhour A, Langhorst J, Benson S, et al. Neural circuitry of abdominal pain-related fear learning and reinstatement in irritable bowel syndrome. Neurogastroenterol Motil 2015;27:114-127.

25. Bouhassira D, Moisset X, Jouet P, Duboc H, Coffin B, Sabate JM. Changes in the modulation of spinal pain processing are related to severity in irritable bowel syndrome. Neurogastroenterol Motil 2013;25:623e468.

26. Lee HF, Hsieh JC, Lu CL, et al. Enhanced affect/cognition-related 
brain responses during visceral placebo analgesia in irritable bowel syndrome patients. Pain 2012;153:1301-1310.

27. Schmid J, Langhorst J, Gaß F, et al. Placebo analgesia in patients with functional and organic abdominal pain: a fMRI study in IBS, UC and healthy volunteers. Gut 2015;64:418-427.

28. Lowén MB, Mayer EA, Sjöberg M, et al. Effect of hypnotherapy and educational intervention on brain response to visceral stimulus in the irritable bowel syndrome. Aliment Pharmcol Ther 2013;37:1184-1197.

29. Labus JS, Mayer EA, Jarcho J, et al. Acute tryptophan depletion alters the effective connectivity of emotional arousal circuitry during visceral stimuli in healthy women. Gut 2011;60:1196-1203.

30. Blankstein U, Chen J, Diamant NE, Davis KD. Altered brain structure in irritable bowel syndrome: potential contributions of pre-existing and disease-driven factors. Gastroenterology 2010;138:1783-1789.

31. Seminowicz DA, Labus JS, Bueller JA, et al. Regional gray matter density changes in brains of patients with irritable bowel syndrome. Gastroenterology 2010;139:48-57, e42.

32. Hong JY, Labus JS, Jiang Z, et al. Regional neuroplastic brain changes in patients with chronic inflammatory and non-inflammatory visceral pain. PLoS One 2014;9:e84564.

33. Jiang Z, Dinov ID, Labus J, et al. Sex-related differences of cortical thickness in patients with chronic abdominal pain. PLoS One 2013;8:e73932.

34. Labus JS, Dinov ID, Jiang Z, et al. Irritable bowel syndrome in female patients is associated with alterations in structural brain networks. Pain 2014;155:137-149.

35. Piché M, Chen JI, Roy M, Poitras P, Bouin M, Rainville P. Thicker posterior insula is associated with disease duration in women with irritable bowel syndrome (IBS) whereas thicker orbitofrontal cortex predicts reduced pain inhibition in both IBS patients and controls. J Pain 2013;14:1217-1226.

36. Berman S, Suyenobu B, Naliboff BD, et al. Evidence for alterations in central noradrenergic signaling in irritable bowel syndrome. Neuroimage 2012;63:1854-1863.

37. Chen JY, Blankstein U, Diamant NE, Davis KD. White matter abnormalities in irritable bowel syndrome and relation to individual factors. Brain Res 2011;1392:121-131.

38. Ellingson BM, Mayer E, Harris RJ, et al. Diffusion tensor imaging detects microstructural reorganization in the brain associated with chronic irritable bowel syndrome. Pain 2013;154:1528-1541.

39. Fang J, Li S, Li M, et al. Altered white matter microstructure identified with tract-based spatial statistics in irritable bowel syndrome: a diffusion tensor imaging study. Brain Imaging Behav 2017;11:1110-1116.

40. Agachan F, Chen T, Pfeifer J, Reissman P, Wexner SD. A constipation scoring system to simplify evaluation and management of constipated patients. Dis Colon Rectum 1996;39:681-685.

41. Li L, Wang HM, Shen Y. Chinese SF-36 health survey: translation, cultural adaptation, validation, and normalisation. J Epidemiol Community Health 2003;57:259-263.

42. Zung WW. A rating instrument for anxiety disorders. Psychosomatics 1971;12:371-379.

43. Zumg WW, Richards CB, Short MJ. Self-rating depression scale in an outpatient clinic. Further validation of the SDS. Arch Gen Psychiatry
$1965 ; 13: 508-515$.

44. Chan AO, Lam KF, Hui WM, et al. Validated questionnaire on diagnosis and symptom severity for functional constipation in the Chinese population. Aliment Pharmacol Ther 2005;22:483-488.

45. Zhou SY, Liu T, Qin HZ, Li Y. [Characteristics of commonly used scales for diagnosis and treatment outcome evaluation in constipation.] Zhongguo Zhong Yao Za Zhi 2013;25:029. [Chinese]

46. Peng H, Zhang YY, Ji Y, Tang WQ, Li Q, Yan XL. Analysis of reliability and validity of Chinese version SDS scale in women of rural area. Chin J Shanghai 2013;14:20-22.

47. Smith SM, Jenkinson M, Johansen-Berg H, et al. Tract-based spatial statistics: voxelwise analysis of multi-subject diffusion data. Neuroimage 2006;31:1487-1505.

48. Mori S, Zhang J. Principles of diffusion tensor imaging and its applications to basic neuroscience research. Neuron 2006;51:527-539.

49. Beaulieu C, Allen PS. Determinants of anisotropic water diffusion in nerves. Magnet Reson Med 1994;31:394-400.

50. Neil JJ, Shiran SI, McKinstry RC, et al. Normal brain in human newborns: apparent diffusion coefficient and diffusion anisotropy measured by using diffusion tensor MR imaging. Radiology 1998;209:57-66.

51. Versace A, Almeida JR, Hassel S, et al. Elevated left and reduced right orbitomedial prefrontal fractional anisotropy in adults with bipolar disorder revealed by tract-based spatial statistics. Arch Gen Psychiat 2008;65:1041-1052.

52. Fenlon LR, Richards LJ. Contralateral targeting of the corpus callosum in normal and pathological brain function. Trends Neurosci 2015;38:264272.

53. Fabri M, Pierpaoli C, Barbaresi P, Polonara G. Functional topography of the corpus callosum investigated by DTI and fMRI. World J Radiol 2014;6:895-906.

54. Kim DJ, Lim M, Kim JS, Son KM, Kim HA, Chung CK. Altered white matter integrity in the corpus callosum in fibromyalgia patients identified by tract-based spatial statistical analysis. Arthritis Rheumatol 2014;66:3190-3199.

55. Yuan K, Qin W, Liu P, et al. Reduced fractional anisotropy of corpus callosum modulates inter-hemispheric resting state functional connectivity in migraine patients without aura. PLoS One 2012;7:e45476.

56. Moayedi M, Weissman-Fogel I, Salomons TV, et al. White matter brain and trigeminal nerve abnormalities in temporomandibular disorder. Pain 2012;153:1467-1477.

57. Horton JE, Crawford HJ, Harrington G, Downs JH 3rd. Increased anterior corpus callosum size associated positively with hypnotizability and the ability to control pain. Brain 2004;127(pt 8):1741-1747.

58. Lim YH, Kim DH, Lee MY, Joo MC. Bowel dysfunction and colon transit time in brain-injured patients. Ann Rehabil Med 2012;36:371378.

59. Ansari R, Sohrabi S, Ghanaie O, et al. Comparison of colonic transit time between patients with constipation-predominant irritable bowel syndrome and functional constipation. Indian J Gastroenterol 2010;29:66-68.

60. Wang X, Pathak S, Stefaneanu L, Yeh FC, Li S, Fernandez-Miranda JC. Subcomponents and connectivity of the superior longitudinal fasciculus in the human brain. Brain Struct Funct 2016;221:2075-2092. 
61. Hurley-Gius KM, Neafsey EJ. The medial frontal cortex and gastric motility: microstimulation results and their possible significance for the overall pattern of organization of rat frontal and parietal cortex. Brain Res 1986;365:241-248.

62. Siah KT, Wong RK, Whitehead WE. Chronic constipation and constipation-predominant IBS: separate and distinct disorders or a spectrum of disease? Gastroenterol Hepatol (N Y) 2016;12:171-178.

63. Wu W. Self-rating anxiety scale (SAS). Shanghai Arch Psychol 1990;2:44.

64. Chunfang W, Zehuan C, Qing X. Self-rating depression scale (SDS): an analysis on 1340 health subjects. Chin J Nerv Men Dis 1986;12:267268.

65. Fukudo S. Stress and visceral pain: focusing on irritable bowel syndrome. Pain 2013;154(suppl 1):S63-S70.

66. Tillisch K, Mayer EA, Labus JS. Quantitative meta-analysis identifies brain regions activated during rectal distension in irritable bowel syndrome. Gastroenterology 2011;140:91-100.

67. Stanghellini V, Tosetti C, Barbara G, et al. Dyspeptic symptoms and gastric emptying in the irritable bowel syndrome. Am J Gastroenterol 2002;97:2738-2743 